\title{
ESTIMATION OF HETEROSIS AND INBREEDING DEPRESSION IN 4 BARLEY UNDER NORMAL AND WATER STRESS CONDITIONS.
}

\author{
R. A. El-Refaey ${ }^{(1)}$, E. H. El-Seidy ${ }^{(1)}$, A.A. El-Gammaal ${ }^{(1)}$, M. Mansour ${ }^{(2)}$ \\ and H.M. Ashry ${ }^{(1)}$ \\ (1) Agronomy Dept., Fac. of Agric., Tanta University \\ (2) Barley Dept., Field Crops Res. Institute, ARC, Egypt
}

Received: Nov. 26, 2016

Accepted: Dec. 3, 2016

\begin{abstract}
The objective of the present investigation was to estimate heterosis and inbreeding depression controlling the agronomic traits and yield and its components of 4 barley crosses, by means of the six populations $\left(P_{1}, P_{2}, F_{1}, F_{2}, B C_{1}\right.$ and $\left.B C_{2}\right)$ of the four barley crosses. Results revealed that, positive heterotic effects relative to the mid parent and better parent were found for most of the studied traits under both conditions. Generally, the most promising crosses were the two crosses 2 and 4, were found to be higher in magnitude, which will be interest in breeding programs for improving the most studied traits in barley.
\end{abstract}

Key words: Barley, heterosis, inbreeding depression.

\section{INTRODUCTION}

Barley (Hordeum vulgare L.) is as ancient as the origin of agriculture itself. The antiquity of barley is documented to periods of 5000 to 7000 B.C or earlier. It is said that barley is the most widely adapted of all grains. It is more tolerant to drought and to saline and alkaline soils than other cereals. Like other cereals it has utility as a feed and food grain, and since ancient times it has been the preferred grain in preparing malt and as a starch source for alcoholic beverages. Its largest use for animal feed. Barley is the world's fourth most important crop, the fourth ranking cereal in the USA and the second ranking cereal in Canada and some other countries.

In Egypt barley is one of the most important winter cereal crops grown mainly in rainfed areas where limited water supply is a feature such as in the Northwest Coastal region and North of Sinai, also grow over wide range of soil variability and under many diverse climatic conditions compared with many other grain crops. So, it can be grown in irrigated saline lands and poor soil conditions. It has also been grown in the newly reclaimed lands and the old ones. In this respect, Katta et al. (2009) and Amer (2010), reported the possibility of developing some barley genotypes combining high yield potential under a wide range of environmental stresses.

Therefore, the main objective of this study included the induction of new promising barley genotypes that are able to produce high yield and are more tolerant to water stress condition.

\section{MATERIALS AND METHODS}

The experimental material comprised four parental varieties of barley based on their variability under drought stress to obtain the following four crosses; cross 1 (Giza 126 x Giza 129); cross 2 (Giza 126 x Giza 131); cross 3 (Giza 131 x Giza 129) and (CC89 x Giza 131). Pedigree of parental genotypes is given in Table (1).

The present study was carried out at the Experimental Farm of Sakha Agricultural Research Station, Kafr El-Sheikh Governorate, Egypt, during the three growing seasons of 2013/14, 2014/15 and 2015/16. In 2013/14 season, the parental genotypes were crossed to obtain the hybrid grains. In 2014/15 season, the hybrid grains of the four crosses were sown to give $F_{1}$ plants, at the same time, these plants were selfed to produce $F_{2}$ and some of $F_{1}$ plants 
of each cross were backcrossed to each of the two parents to produce the two backcrosses $\left(\mathrm{BC}_{1}\right.$ and $\left.\mathrm{BC}_{2}\right)$, at the same time the crosses were made again between the parents of each cross to obtain enough $F_{1}$ grains. In 2015/16 season, the basic generation $\left(P_{1}, P_{2}, F_{1}, F_{2}, B C_{1}\right.$ and $\left.B_{2}\right)$ of the four crosses were sown in a randomized complete blocks design with three replications under normal (three irrigation after sowing; irrigation at tillering, at elongation and at heading stage (favorable condition)) and water stress condition (sowing irrigation only). There was three rows for each of $P_{1}, P_{2}$ and $F_{1}$ generation, seven rows for each of $\mathrm{BC}_{1}, \mathrm{BC}_{2}$ and twenty five rows for the $F_{2}$ generation. Row was $1.5 \mathrm{~m}$ in length, $30 \mathrm{~cm}$. apart and $15 \mathrm{~cm}$. between grains within a row. Data were recorded on 30, 30, 300 and 75 plants were selected at random for both parents, $F_{1}, F_{2}$ and backcrosses of each cross.

The amount of irrigation water supplied and total rainfall in $\mathrm{m}^{3} / \mathrm{fad}^{*}$ for different treatments are presented in Table (2). Monthly mean air temperature, mean relative humidity and rainfall ( $\mathrm{mm} / \mathrm{month})$ in winter season of $2015 / 16$ at experimental site are presented in Table (3).

Table (1): Name, pedigree, origin and degree of drought tolerance of four barley genotypes.

\begin{tabular}{|c|c|c|c|c|}
\hline No. & Genotypes & Pedigree & Origin & $\begin{array}{l}\text { drought } \\
\text { tolerance }\end{array}$ \\
\hline 1 & Giza 126 & BaladiBahteem/SD729-por12762-Bc & $\begin{array}{l}\text { Local variety } \\
\text { (Egypt) }\end{array}$ & $\mathrm{T}$ \\
\hline 2 & Giza 129 & Deir Alla 106/Cel//As46/Aths*2 & $\begin{array}{l}\text { Local variety } \\
\text { (Egypt }\end{array}$ & S \\
\hline 3 & Giza 131 & $\begin{array}{l}\text { CM67-B/CENTENO//CAM-B/3/ROW906.73 /4 / } \\
\text { GLORIA-BAR/COME-B/5/ FALCON -BAR /6/ } \\
\text { LINO }\end{array}$ & $\begin{array}{l}\text { Local variety } \\
\text { (Egypt }\end{array}$ & $\mathrm{T}$ \\
\hline 4 & CC 89 & $\begin{array}{l}\text { Panniy/Salmas/5/Baca"s"/3/AC253//Cl08887/CI0 } \\
\text { 5761/4/JLB70-01 }\end{array}$ & ICARDA & S \\
\hline
\end{tabular}

Tolerant (T), Sensitive (S)

Table (2): Amount of irrigation water supplied and total rainfall in $\mathrm{m}^{3} / \mathrm{fed}$ for different treatments.

\begin{tabular}{|c|c|c|c|c|c|c|c||}
\hline \multirow{2}{*}{ Treatment } & \multicolumn{3}{|c|}{ water applied $\left(\mathrm{m}^{3} / \mathrm{fad}\right)$ at } & & Total \\
\cline { 2 - 7 } & Sowing & II & III & VI & $\begin{array}{c}\text { Total } \\
\text { irrigation, } \\
\left(\mathrm{m}^{3} / \mathrm{fad}\right)\end{array}$ & $\begin{array}{c}\text { Rainfall, } \\
\left(\mathrm{m}^{3} / \mathrm{fad}\right)\end{array}$ & \\
\hline T1 (normal) & 550 & 221.6 & 340.8 & 378 & 1490.4 & 84.21 & 1574.61 \\
\hline T 2 (stress) & 550 & - & - & - & 550 & 84.21 & 634.21 \\
\hline
\end{tabular}

* Fadden (fad.) $=4200 \mathrm{~m}^{2}$ 
Table (3): Monthly mean air temperature $\left(\mathrm{C}^{\circ}\right)$, mean relative humidity $(\mathrm{RH} \%)$ and rainfall ( $\mathrm{mm} / \mathrm{month}$ ) in winter season of $2015 / 16$ at Sakha site.

\begin{tabular}{|c|c|c|c|c|c|}
\hline \multirow{2}{*}{\multicolumn{2}{|c|}{ Months }} & \multicolumn{2}{|c|}{ Temperature $\left(C^{0}\right)$} & \multirow{3}{*}{$\begin{array}{l}\mathrm{RH} \% \\
77.23\end{array}$} & \multirow{3}{*}{$\begin{array}{c}\begin{array}{c}\text { Rainfall } \\
(\mathrm{mm})\end{array} \\
22.35\end{array}$} \\
\hline & & maximum & minimum & & \\
\hline \multirow{2}{*}{ December, 2015} & $(1-15)$ & 18.66 & 8.6 & & \\
\hline & $(16-31)$ & 18.88 & 8.1 & 77.20 & 2.65 \\
\hline \multirow{2}{*}{ January, 2016} & $(1-15)$ & 19.65 & 6.98 & 76.60 & 21.46 \\
\hline & $(16-31)$ & 18.01 & 5.84 & 75.47 & 24.55 \\
\hline \multirow{2}{*}{ February, 2016} & $(1-15)$ & 21.49 & 8.68 & 48.9 & 0.00 \\
\hline & $(16-28)$ & 24.51 & 10.66 & 67.7 & 0.00 \\
\hline \multirow{2}{*}{ March, 2016} & $(1-15)$ & 25.65 & 11.75 & 73.46 & 10.70 \\
\hline & $(16-31)$ & 22.81 & 11.57 & 71.6 & 2.50 \\
\hline \multirow{2}{*}{ April, 2016} & $(1-15)$ & 28.29 & 17.88 & 64.86 & 0.00 \\
\hline & $(16-31)$ & 31.21 & 19.94 & 58.76 & 0.00 \\
\hline \multirow{2}{*}{ May, 2016} & $(1-15)$ & 32.35 & 23.21 & 58.7 & 0.00 \\
\hline & $(16-31)$ & 29.44 & 23.07 & 59.3 & 0.00 \\
\hline
\end{tabular}

*Sakha Agric. Res. Station, Egypt.

The data were collected on the basis of individual plant for days to heading, days to maturity, grain filling period, grain filling rate, plant height, spike length, $\overline{p e d u n c l e ~}$ length, number of tillers, number of spikes per plant, number of grains per spike, grains weight per spike 100-grain weight, biological yield per plant and grain yield per plant. The growing conditions were identical for all the six generations followed by the standard practices.

\section{Statistical and genetic analysis:}

Heterosis was calculated as the deviation of $F_{1}$ mean from the mid-parent and better parent values and expressed in percentage according to Mather and Jinks (1982). Inbreeding depression was calculated as the difference between the $F_{1}$ and $F_{2}$ means as a percentage of $F_{1}$. The "t" test was used to determine the significance of these deviations where the standard error (SE) was calculated as follows:

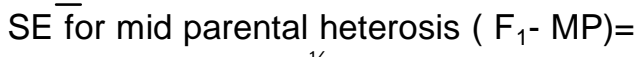
$\left(\mathrm{V}_{\mathrm{F} 1}+1 / 4 \mathrm{~V}_{\mathrm{P} 2}+1 / 4 \mathrm{~V}_{\mathrm{P} 2}\right)^{1 / 2}$

$\mathrm{SE}$ for better parental heterosis $\left(\mathrm{F}_{1^{-}}\right.$ $\mathrm{BP})=\left(\mathrm{V}_{\mathrm{F} 1}+\mathrm{V}_{\mathrm{BP}}\right)^{1 / 2}$

SE for inbreeding depression $\left(F_{1}-F_{2}\right)=$ $\left(V_{F 1}+V_{F 2}\right)^{1 / 2}$

where, the $t$ is the deviation/SE at the corresponding degrees of freedom.

Simth (1952) proposed the following equation to determine potence ratio $(P)$, $\mathrm{P}=\overline{\mathrm{F}_{1}}-\mathrm{MP} \overline{\mathrm{P} /}{ }_{2}^{1 /}\left(\mathrm{P}_{1}-\mathrm{P}_{2}\right)$ which can be defined as follows:

Where,

$\left(F_{1}\right)$ first generation mean, $\left(P_{1}\right)$ the mean of the largest parent, $\left(P_{2}\right)$ the mean of the smallest parent and (MP) mid-parent value.

\section{RESULTS AND DISCUSSION Mean and variance of mean:}

Mean and variance of mean for the studied traits in the four crosses for six populations $\mathrm{P}_{1}, \mathrm{P}_{2}, \mathrm{~F}_{1}, \mathrm{~F}_{2}, \mathrm{BC}_{1}$ and $\mathrm{BC}_{2}$ under two conditions of irrigation are presented in Tables (4 a-e). These data 
were used to calculate the scalling test and six parameters as Gamble (1962) procedure.

Analysis of variance indicted significant differences among generations in all traits under this study. The results revealed that, generation means decreased under stress than in normal and significantly different for all the studied traits in all crosses towards earliness for heading, maturity and grain filling period, except for grain yield/plant in the $\mathrm{P}_{1}$ of the third cross. Amer (2011) found that mean performance was decreased significantly under stress than normal condition. The $F_{1}$ mean values exceeded the mid values of the two parental means for most of the studied traits in the four crosses under both conditions. The $F_{2}$ population mean performance values were intermediate between the two parents and less than $F_{1}$ mean performance values for grain yield and its components under normal irrigation and water stress conditions. However, the two populations $\left(\mathrm{BC}_{1}\right.$ and $\left.\mathrm{BC}_{2}\right)$ mean performance values varied under the two irrigation treatments and each trait tended toward the mean of its recurrent parent. These results agreed with those obtained by El-Sayed (2007) and El-Shawy (2008).

\section{Heterosis and inbreeding depression:}

Heterosis percent, potence ratio and inbreeding depression for all studied traits in the four crosses are presented in Tables (5 $a-b)$.

In self - pollinated crops such as barley, plant breeders have been investigated the possibility of developing hybrid cultivars. Thus, the utilization of heterosis in various crops overall the world has tremendously increased the production either for human food or livestock feed. Heterosis is a complex phenomenon which depends on the balance of different combinations of genotypic effect as well as the distribution of plus or minus alleles in parents.

Heterosis is expressed as the percentage deviation of $F_{1}$ mean performance from the mid parent or better parent of the trait. High positive values of heterosis would be of interest for most traits under investigation, however, for days to heading, days to maturity and grain filling period, negative values would be of value from the barley breeders point of view, either by producing early mature cultivars to be used in case of intensive agricultural rotation and to make barley plants evading unfavorable weather conditions and disease infection.

For earliness (days to heading, maturity and grain filling period), highly significant negative desirable heterotic effects over mid and better parent were detected in the fourth cross (CC89 $x$ Giza 131) under both conditions and in the second and the third crosses (Giza 126 x Giza 131) and (Giza 131 x Giza 129), respectively under normal condition for days to heading. However, over-dominance (PR $>-1$ ) was responsible for such heterosis in all cases. Moreover, mid-prent heterosis was highly significant in the third cross for days to maturity and grain filling period under both conditions and in the first cross for days to maturity at stress condition, as a result of partial dominance $(P R<-1)$. El-Bawab (2003), Eid (2006), El-Shawy (2008), Khattab et al. (2010), El-Akhdar (2011) and Mohamed (2014) found that the heterotic effect for days to $50 \%$ flowering was more affected by overdominance. While, El-Bawab (2003) found significant negative heterotic effect for maturity date.

The first, the second and the fourth crosses gave highly significant positive heterotic effects over mid and better parent under both condition for grain 
El-Refaey, et al.,

filling rate a result of over- dominance in all cases, except for the third cross under normal condition due to partial dominance $(\mathrm{PR}<+1)$ Meanwhile, the third cross (Giza 131 x Giza 129) under

normal condition showed highly significant positive heterotic effects relative to mid parent, where partial dominance was existed.

Table (4 -a ): Mean $\left(X^{-}\right)$, variance $\left(s^{2}\right)$ and variance of mean $\left(S^{2} x^{-}\right)$of $P_{1}, P_{2}, F_{1}, F_{2}, B C_{1}$ and $\mathrm{BC}_{2}$ populations of four crosses for days to heading, days to maturity, grain filling period and filling rate under normal and water stress conditions.

\begin{tabular}{|c|c|c|c|c|c|c|c|c|c|c|c|c|c|c|}
\hline \multirow{2}{*}{ Traits } & \multirow{2}{*}{ Crosses } & \multirow{2}{*}{$\begin{array}{l}\text { Statistical } \\
\text { Parameter }\end{array}$} & \multicolumn{6}{|c|}{ Normal } & \multicolumn{6}{|c|}{ stress } \\
\hline & & & $P_{1}$ & $\mathrm{P}_{2}$ & $F_{1}$ & $\mathrm{~F}_{2}$ & $\mathrm{BC}_{1}$ & $\mathrm{BC}_{2}$ & $P_{1}$ & $\mathrm{P}_{2}$ & $F_{1}$ & $\mathrm{~F}_{2}$ & $\mathrm{BC}_{1}$ & $\mathrm{BC}_{2}$ \\
\hline \multirow{12}{*}{$\begin{array}{l}\text { Days to } \\
\text { heading } \\
\text { (day) }\end{array}$} & & $\mathrm{X}^{-}$ & 83.90 & 85.20 & 86.20 & 85.72 & 81.48 & 86.79 & 80.00 & 82.70 & 84.10 & 81.24 & 79.85 & 82.04 \\
\hline & & $S^{2}$ & 0.51 & 0.37 & 0.37 & 20.11 & 16.71 & 16.22 & 0.62 & 0.63 & 0.51 & 22.63 & 16.69 & 13.36 \\
\hline & & $S^{2} x$ & 0.02 & 0.01 & 0.01 & 0.07 & 0.22 & 0.22 & 0.02 & 0.02 & 0.02 & 0.08 & 0.22 & 0.18 \\
\hline & \multirow{3}{*}{2} & $x^{-}$ & 83.90 & 85.30 & 82.30 & 81.52 & 79.57 & 80.24 & 80.20 & 79.90 & 80.70 & 77.70 & 83.79 & 81.57 \\
\hline & & $S^{2}$ & 0.51 & 0.42 & 0.42 & 17.59 & 10.68 & 14.83 & 0.58 & 0.30 & 0.63 & 17.17 & 10.63 & 13.00 \\
\hline & & $S^{2} x$ & 0.02 & 0.01 & 0.01 & 0.06 & 0.14 & 0.20 & 0.02 & 0.01 & 0.02 & 0.06 & 0.14 & 0.17 \\
\hline & \multirow{3}{*}{3} & $x^{-}$ & 85.60 & 85.10 & 82.70 & 83.32 & 82.88 & 84.83 & 80.40 & 82.80 & 82.00 & 80.47 & 82.53 & 84.91 \\
\hline & & $S^{2}$ & 0.46 & 0.51 & 0.29 & 19.97 & 18.89 & 14.90 & 0.46 & 0.58 & 0.76 & 27.64 & 14.33 & 24.44 \\
\hline & & $S^{2} x$ & 0.02 & 0.02 & 0.01 & 0.07 & 0.25 & 0.20 & 0.02 & 0.02 & 0.03 & 0.09 & 0.19 & 0.33 \\
\hline & \multirow{3}{*}{4} & $\mathrm{X}^{-}$ & 83.90 & 85.10 & 81.00 & 81.32 & 79.88 & 85.12 & 78.30 & 80.00 & 76.80 & 80.85 & 80.07 & 84.84 \\
\hline & & $S^{2}$ & 0.51 & 0.30 & 0.62 & 29.72 & 22.67 & 20.76 & 0.22 & 0.41 & 0.79 & 23.71 & 11.50 & 19.62 \\
\hline & & $s^{2} x$ & 0.02 & 0.01 & 0.02 & 0.10 & 0.30 & 0.28 & 0.01 & 0.01 & 0.03 & 0.08 & 0.15 & 0.26 \\
\hline \multirow{12}{*}{$\begin{array}{l}\text { Days to } \\
\text { maturity } \\
\text { (day) }\end{array}$} & & $\mathrm{X}^{-}$ & 119.17 & 114.47 & 121.27 & 117.88 & 120.49 & 116.67 & 117.50 & 113.50 & 114.80 & 115.85 & 115.80 & 115.50 \\
\hline & & $S^{2}$ & 0.63 & 0.81 & 0.75 & 12.39 & 10.23 & 10.14 & 0.47 & 0.50 & 0.58 & 6.37 & 4.44 & 5.64 \\
\hline & & $S^{2} x$ & 0.02 & 0.03 & 0.03 & 0.04 & 0.14 & 0.14 & 0.02 & 0.02 & 0.02 & 0.02 & 0.06 & 0.08 \\
\hline & & $\mathrm{X}^{-}$ & 119.23 & 116.63 & 121.33 & 117.63 & 118.69 & 118.88 & 117.70 & 115.80 & 117.10 & 115.33 & 116.83 & 116.80 \\
\hline & & $S^{2}$ & 0.67 & 0.79 & 0.85 & 10.04 & 7.76 & 9.05 & 0.49 & 0.62 & 0.30 & 9.01 & 8.01 & 6.72 \\
\hline & & $S^{2} x$ & 0.02 & 0.03 & 0.03 & 0.03 & 0.10 & 0.12 & 0.02 & 0.02 & 0.01 & 0.03 & 0.11 & 0.09 \\
\hline & & $x^{-}$ & 118.07 & 114.20 & 115.00 & 116.13 & 117.61 & 115.08 & 117.07 & 113.50 & 114.50 & 114.95 & 114.43 & 113.80 \\
\hline & & $S^{2}$ & 0.62 & 0.65 & 0.83 & 8.12 & 6.29 & 6.91 & 0.55 & 0.28 & 0.47 & 6.47 & 4.81 & 5.41 \\
\hline & & $S^{2} x$ & 0.02 & 0.02 & 0.03 & 0.03 & 0.08 & 0.09 & 0.02 & 0.01 & 0.02 & 0.02 & 0.06 & 0.07 \\
\hline & & $x^{-}$ & 118.03 & 116.53 & 115.30 & 116.24 & 117.32 & 117.84 & 116.57 & 115.30 & 114.37 & 115.67 & 115.47 & 115.53 \\
\hline & & $s^{2}$ & 0.72 & 0.81 & 0.56 & 7.44 & 5.22 & 6.19 & 0.60 & 0.68 & 0.52 & 6.26 & 4.40 & 5.29 \\
\hline & & $S^{2} x^{-}$ & 0.02 & 0.03 & 0.02 & 0.02 & 0.07 & 0.08 & 0.02 & 0.02 & 0.02 & 0.02 & 0.06 & 0.07 \\
\hline \multirow{12}{*}{$\begin{array}{l}\text { Grain } \\
\text { filling } \\
\text { period }\end{array}$} & & $\mathrm{X}^{-}$ & 39.40 & 31.40 & 37.17 & 34.38 & 40.84 & 34.63 & 37.50 & 30.80 & 30.70 & 32.04 & 35.71 & 34.03 \\
\hline & & $S^{2}$ & 1.14 & 1.28 & 2.07 & 27.07 & 23.60 & 21.37 & 0.88 & 0.79 & 1.04 & 25.79 & 22.80 & 20.24 \\
\hline & & $S^{2} x^{-}$ & 0.04 & 0.04 & 0.07 & 0.09 & 0.31 & 0.28 & 0.03 & 0.03 & 0.03 & 0.09 & 0.30 & 0.27 \\
\hline & & $x^{-}$ & 39.00 & 36.60 & 40.80 & 37.78 & 36.07 & 37.67 & 37.70 & 35.60 & 36.20 & 35.82 & 33.08 & 35.29 \\
\hline & 2 & $\mathrm{~S}^{2}$ & 1.24 & 1.49 & 1.61 & 26.44 & 21.74 & 23.04 & 1.04 & 0.87 & 1.20 & 25.60 & 20.02 & 21.48 \\
\hline & & $S^{2} x^{-}$ & 0.04 & 0.05 & 0.05 & 0.09 & 0.29 & 0.31 & 0.03 & 0.03 & 0.04 & 0.09 & 0.27 & 0.29 \\
\hline & & $\mathrm{X}^{-}$ & 37.90 & 31.70 & 33.00 & 35.01 & 35.08 & 30.29 & 36.20 & 30.77 & 32.47 & 32.81 & 31.81 & 29.57 \\
\hline & 3 & $S^{2}$ & 1.54 & 1.87 & 1.59 & 33.59 & 25.34 & 27.70 & 1.27 & 1.50 & 0.74 & 28.10 & 23.26 & 25.95 \\
\hline & & $S^{2} x^{-}$ & 0.05 & 0.06 & 0.05 & 0.11 & 0.34 & 0.37 & 0.04 & 0.05 & 0.02 & 0.09 & 0.31 & 0.35 \\
\hline & & $\mathrm{X}^{-}$ & 40.20 & 36.50 & 34.77 & 34.76 & 40.45 & 32.72 & 38.07 & 35.50 & 33.60 & 34.76 & 34.01 & 31.36 \\
\hline & 4 & $S^{2}$ & 1.06 & 1.71 & 2.05 & 35.58 & 27.31 & 26.93 & 0.75 & 1.29 & 1.70 & 29.05 & 25.36 & 23.99 \\
\hline & & $S^{2} x^{-}$ & 0.04 & 0.06 & 0.07 & 0.12 & 0.36 & 0.36 & 0.03 & 0.04 & 0.06 & 0.10 & 0.34 & 0.32 \\
\hline & & $\mathrm{X}^{-}$ & 0.58 & 0.76 & 0.95 & 0.46 & 0.64 & 0.59 & 0.52 & 0.54 & 0.94 & 0.50 & 0.45 & 0.60 \\
\hline & 1 & $S^{2}$ & 0.001 & 0.004 & 0.003 & 0.066 & 0.040 & 0.059 & 0.002 & 0.007 & 0.008 & 0.098 & 0.067 & 0.069 \\
\hline Grain & & $S^{2} x^{-}$ & 0.0003 & 0.0001 & 0.0001 & 0.0002 & 0.0005 & 0.0007 & 0.0006 & 0.0002 & 0.0002 & 0.0003 & 0.0009 & 0.0009 \\
\hline & & $x^{-}$ & 0.79 & 0.77 & 1.13 & 0.69 & 0.75 & 0.67 & 0.67 & 0.61 & 0.82 & 0.49 & 0.54 & 0.67 \\
\hline & 2 & $S^{2}$ & 0.002 & 0.004 & 0.005 & 0.077 & 0.056 & 0.052 & 0.004 & 0.005 & 0.006 & 0.089 & 0.066 & 0.073 \\
\hline & & $S^{2} x$ & 0.0006 & 0.0001 & 0.0001 & 0.0002 & 0.0007 & 0.0006 & 0.0001 & 0.0001 & 0.0002 & 0.0002 & 0.0008 & 0.0009 \\
\hline & & $\mathrm{X}^{-}$ & 1.15 & 0.63 & 1.01 & 0.74 & 0.97 & 0.71 & 0.94 & 0.40 & 0.38 & 0.58 & 0.59 & 0.61 \\
\hline & 3 & $S^{2}$ & 0.01 & 0.01 & 0.005 & 0.10 & 0.07 & 0.07 & 0.004 & 0.005 & 0.006 & 0.091 & 0.082 & 0.059 \\
\hline & & $S^{2} x^{-}$ & 0.0003 & 0.0003 & 0.0001 & 0.0003 & 0.0009 & 0.0009 & 0.0001 & 0.0001 & 0.0002 & 0.0003 & 0.001 & 0.0007 \\
\hline & & $x^{-}$ & 0.59 & 0.89 & 0.94 & 0.85 & 0.62 & 0.73 & 0.46 & 0.62 & 0.66 & 0.84 & 0.65 & 0.64 \\
\hline & 4 & $S^{2}$ & 0.002 & 0.005 & 0.007 & 0.092 & 0.058 & 0.069 & 0.003 & 0.004 & 0.008 & 0.105 & 0.075 & 0.077 \\
\hline & & $S^{2} x^{-}$ & 0.0006 & 0.0001 & 0.0002 & 0.0003 & 0.0007 & 0.0009 & 0.0001 & 0.0001 & 0.0002 & 0.0003 & 0.001 & 0.001 \\
\hline
\end{tabular}

Cross 1 (Giza $126 \times$ Giza 129), Cross2 (Giza 126 x Giza 131), Cross 3 (Giza $131 \times$ Giza 129) and cross 4 (CC89 x Giza131) 
Estimation of heterosis and inbreeding depression in 4 barley under ...

Table (4-b): Mean $\left(X^{-}\right)$, variance $\left(s^{2}\right)$ and variance of mean $\left(\left(S^{2}{ }^{-}\right)\right.$of $P_{1}, P_{2}, F_{1}, F_{2}, B C_{1}$ and $\mathrm{BC}_{2}$ populations of four crosses for plant height, spike length, peduncle length and number of tillers/ plant under normal and water tress conditions.

\begin{tabular}{|c|c|c|c|c|c|c|c|c|c|c|c|c|c|c|}
\hline \multirow{2}{*}{ Traits } & \multirow{2}{*}{ Crosses } & \multirow{2}{*}{$\begin{array}{l}\text { Statistical } \\
\text { Parameter }\end{array}$} & \multicolumn{6}{|c|}{ Normal } & \multicolumn{6}{|c|}{ stress } \\
\hline & & & $P_{1}$ & $P_{2}$ & $F_{1}$ & $\mathrm{~F}_{2}$ & $\mathrm{BC}_{1}$ & $\mathrm{BC}_{2}$ & $P_{1}$ & $P_{2}$ & $\mathrm{~F}_{1}$ & $\mathrm{~F}_{2}$ & $\mathrm{BC}_{1}$ & $\mathrm{BC}_{2}$ \\
\hline \multirow{12}{*}{$\begin{array}{l}\text { Plant } \\
\text { height } \\
(\mathrm{cm})\end{array}$} & \multirow{3}{*}{1} & $\mathrm{X}^{-}$ & 99.20 & 101.80 & 102.10 & 100.28 & 96.84 & 98.89 & 98.50 & 99.70 & 101.10 & 100.77 & 93.84 & 98.20 \\
\hline & & $S^{2}$ & 2.44 & 5.13 & 4.44 & 68.66 & 60.22 & 53.61 & 13.91 & 2.08 & 2.58 & 84.77 & 69.43 & 70.22 \\
\hline & & $S^{2} x$ & 0.08 & 0.17 & 0.15 & 0.23 & 0.80 & 0.71 & 0.46 & 0.07 & 0.09 & 0.28 & 0.93 & 0.94 \\
\hline & \multirow{3}{*}{2} & $\mathrm{X}^{-}$ & 98.70 & 102.50 & 106.90 & 105.29 & 103.25 & 103.71 & 97.50 & 96.40 & 105.60 & 104.14 & 93.20 & 96.79 \\
\hline & & $S^{2}$ & 6.42 & 4.60 & 5.47 & 67.69 & 58.35 & 57.18 & 4.19 & 13.08 & 7.70 & 77.98 & 63.76 & 62.25 \\
\hline & & $S^{2} x^{-}$ & 0.21 & 0.15 & 0.18 & 0.23 & 0.78 & 0.76 & 0.14 & 0.44 & 0.26 & 0.26 & 0.85 & 0.83 \\
\hline & \multirow{3}{*}{3} & $\mathrm{X}^{-}$ & 100.40 & 100.00 & 103.90 & 101.35 & 95.37 & 102.57 & 98.90 & 98.10 & 100.80 & 99.55 & 94.21 & 86.92 \\
\hline & & $S^{2}$ & 18.87 & 15.93 & 13.33 & 132.77 & 110.59 & 118.25 & 8.58 & 8.16 & 9.27 & 100.78 & 85.93 & 92.56 \\
\hline & & $S^{2} x^{-}$ & 0.63 & 0.53 & 0.44 & 0.44 & 1.47 & 1.58 & 0.29 & 0.27 & 0.31 & 0.34 & 1.15 & 1.23 \\
\hline & \multirow{3}{*}{4} & $x^{-}$ & 103.70 & 103.00 & 104.30 & 103.19 & 99.33 & 102.87 & 98.00 & 98.70 & 103.20 & 101.77 & 93.80 & 100.53 \\
\hline & & $S^{2}$ & 4.77 & 3.72 & 3.73 & 59.04 & 49.93 & 48.52 & 9.72 & 7.87 & 3.89 & 128.43 & 106.81 & 98.85 \\
\hline & & $S^{2} x$ & 0.16 & 0.12 & 0.12 & 0.20 & 0.67 & 0.65 & 0.32 & 0.26 & 0.13 & 0.43 & 1.42 & 1.32 \\
\hline \multirow{12}{*}{$\begin{array}{l}\text { Spike } \\
\text { length } \\
(\mathrm{cm})\end{array}$} & \multirow{3}{*}{1} & $\mathrm{X}^{-}$ & 7.60 & 8.50 & 10.40 & 9.62 & 8.04 & 8.22 & 6.50 & 7.20 & 9.50 & 7.02 & 7.83 & 7.41 \\
\hline & & $S^{2}$ & 0.25 & 0.26 & 0.25 & 2.86 & 2.07 & 2.36 & 0.26 & 0.17 & 0.26 & 3.17 & 2.36 & 2.66 \\
\hline & & $S^{2} x^{-}$ & 0.01 & 0.01 & 0.01 & 0.01 & 0.03 & 0.03 & 0.01 & 0.01 & 0.01 & 0.01 & 0.03 & 0.04 \\
\hline & \multirow{3}{*}{ ? } & $x^{-}$ & 7.70 & 9.87 & 10.30 & 8.42 & 9.57 & 8.57 & 7.20 & 6.60 & 9.70 & 8.09 & 8.88 & 8.70 \\
\hline & & $S^{2}$ & 0.22 & 0.19 & 0.22 & 2.95 & 1.98 & 2.30 & 0.17 & 0.25 & 0.22 & 2.91 & 2.32 & 1.97 \\
\hline & & $S^{2} x$ & 0.01 & 0.01 & 0.01 & 0.01 & 0.03 & 0.03 & 0.01 & 0.01 & 0.01 & 0.01 & 0.03 & 0.03 \\
\hline & \multirow{3}{*}{3} & $x^{-}$ & 10.63 & 10.67 & 11.60 & 9.82 & 10.16 & 9.91 & 9.50 & 9.33 & 10.47 & 9.51 & 9.08 & 9.80 \\
\hline & & $S^{2}$ & 0.24 & 0.23 & 0.25 & 1.69 & 1.33 & 1.16 & 0.26 & 0.23 & 0.26 & 1.62 & 1.45 & 1.10 \\
\hline & & $S^{2} x^{-}$ & 0.01 & 0.01 & 0.01 & 0.01 & 0.02 & 0.02 & 0.01 & 0.01 & 0.01 & 0.01 & 0.02 & 0.01 \\
\hline & \multirow{3}{*}{4} & $x^{-}$ & 7.63 & 10.30 & 11.50 & 9.36 & 8.09 & 9.08 & 7.40 & 9.60 & 10.70 & 8.88 & 7.23 & 8.04 \\
\hline & & $S^{2}$ & 0.24 & 0.22 & 0.26 & 2.28 & 1.71 & 1.83 & 0.25 & 0.25 & 0.22 & 2.88 & 1.91 & 2.18 \\
\hline & & $S^{2} x^{-}$ & 0.01 & 0.01 & 0.01 & 0.01 & 0.02 & 0.02 & 0.01 & 0.01 & 0.01 & 0.01 & 0.03 & 0.03 \\
\hline \multirow{12}{*}{$\begin{array}{l}\text { Peduncle } \\
\text { Length - } \\
\text { (cm) }\end{array}$} & \multirow{3}{*}{1} & $\mathrm{X}^{-}$ & 26.10 & 24.60 & 33.20 & 24.27 & 28.81 & 24.17 & 22.80 & 18.40 & 23.50 & 22.34 & 22.08 & 20.00 \\
\hline & & $S^{2}$ & 1.33 & 1.08 & 1.20 & 16.75 & 16.37 & 14.69 & 1.41 & 1.28 & 0.88 & 17.46 & 14.18 & 13.92 \\
\hline & & $S^{2} x^{-}$ & 0.04 & 0.04 & 0.04 & 0.06 & 0.22 & 0.20 & 0.05 & 0.04 & 0.03 & 0.06 & 0.19 & 0.19 \\
\hline & & $\mathrm{X}^{-}$ & 29.80 & 27.80 & 33.60 & 32.03 & 25.47 & 31.27 & 26.00 & 27.00 & 29.70 & 26.58 & 26.32 & 27.44 \\
\hline & 2 & $S^{2}$ & 0.99 & 1.20 & 1.28 & 27.54 & 19.06 & 21.85 & 1.45 & 1.45 & 1.25 & 22.38 & 19.87 & 15.14 \\
\hline & & $S^{2} x^{-}$ & 0.03 & 0.04 & 0.04 & 0.09 & 0.25 & 0.29 & 0.05 & 0.05 & 0.04 & 0.07 & 0.26 & 0.20 \\
\hline & & $\mathrm{X}^{-}$ & 25.20 & 23.50 & 29.30 & 24.79 & 25.72 & 25.32 & 23.30 & 22.10 & 27.20 & 23.03 & 25.45 & 23.01 \\
\hline & 3 & $S^{2}$ & 0.99 & 0.88 & 1.25 & 29.72 & 29.91 & 20.82 & 1.04 & 1.13 & 1.41 & 18.22 & 17.36 & 11.26 \\
\hline & & $S^{2} x$ & 0.03 & 0.03 & 0.04 & 0.10 & 0.40 & 0.28 & 0.03 & 0.04 & 0.05 & 0.06 & 0.23 & 0.15 \\
\hline & & $X^{-}$ & 26.40 & 28.90 & 31.70 & 30.03 & 26.61 & 29.88 & 23.40 & 26.80 & 27.40 & 25.56 & 25.23 & 27.59 \\
\hline & 4 & $S^{2}$ & 0.46 & 1.33 & 1.04 & 30.53 & 24.29 & 25.08 & 1.49 & 0.99 & 1.70 & 27.86 & 13.15 & 18.79 \\
\hline & & $S^{2} x$ & 0.02 & 0.04 & 0.03 & 0.10 & 0.32 & 0.33 & 0.05 & 0.03 & 0.06 & 0.09 & 0.18 & 0.25 \\
\hline & & $\mathrm{X}^{-}$ & 21.50 & 19.40 & 23.80 & 20.84 & 17.20 & 18.72 & 9.70 & 17.80 & 18.50 & 11.80 & 10.64 & 14.19 \\
\hline & 1 & $S^{2}$ & 2.12 & 1.08 & 1.20 & 38.32 & 26.59 & 30.10 & 2.91 & 1.20 & 1.71 & 25.06 & 20.02 & 19.99 \\
\hline & & $S^{2} x$ & 0.07 & 0.04 & 0.04 & 0.13 & 0.35 & 0.40 & 0.10 & 0.04 & 0.06 & 0.08 & 0.27 & 0.27 \\
\hline of tillers/ & & $\mathrm{X}^{-}$ & 21.10 & 17.40 & 25.80 & 22.34 & 17.92 & 23.19 & 15.30 & 14.20 & 17.00 & 16.77 & 16.52 & 18.63 \\
\hline plant & 2 & $S^{2}$ & 1.54 & 2.32 & 1.82 & 83.36 & 57.16 & 67.32 & 1.04 & 1.61 & 1.03 & 14.69 & 12.50 & 11.51 \\
\hline & & $S^{2} x^{-}$ & 0.05 & 0.08 & 0.06 & 0.28 & 0.76 & 0.90 & 0.03 & 0.05 & 0.03 & 0.05 & 0.17 & 0.15 \\
\hline & & $\mathrm{X}^{-}$ & 23.70 & 17.40 & 24.60 & 18.41 & 21.80 & 14.99 & 17.50 & 14.60 & 14.40 & 11.68 & 14.00 & 10.65 \\
\hline & 3 & $S^{2}$ & 2.70 & 3.14 & 1.70 & 81.75 & 64.30 & 72.85 & 1.71 & 1.28 & 1.70 & 22.31 & 21.32 & 19.50 \\
\hline & & $S^{2} x^{-}$ & 0.09 & 0.10 & 0.06 & 0.27 & 0.86 & 0.97 & 0.06 & 0.04 & 0.06 & 0.07 & 0.28 & 0.26 \\
\hline & & $\mathrm{X}^{-}$ & 20.50 & 18.10 & 21.10 & 19.13 & 23.00 & 18.48 & 17.40 & 17.30 & 20.60 & 18.15 & 19.16 & 16.37 \\
\hline & 4 & $S^{2}$ & 2.53 & 1.75 & 1.75 & 61.74 & 54.24 & 50.25 & 3.35 & 4.98 & 1.90 & 43.09 & 37.51 & 35.48 \\
\hline & & $S^{2} x^{-}$ & 0.08 & 0.06 & 0.06 & 0.21 & 0.72 & 0.67 & 0.11 & 0.17 & 0.06 & 0.14 & 0.50 & 0.47 \\
\hline
\end{tabular}

Cross 1 (Giza $126 \times$ Giza 129), Cross2 (Giza $126 \times$ Giza 131), Cross 3 (Giza $131 \times$ Giza 129) and cross 4 (CC89 x Giza131) 
El-Refaey, et al.,

Table (4-c ): Mean $\left(X^{-}\right)$, variance $\left(s^{2}\right)$ and variance of mean $\left(S^{2} x^{-}\right)$of $P_{1}, P_{2}, F_{1}, F_{2}, B C_{1}$ and $\mathrm{BC}_{2}$ populations of four crosses for number of spike/ plant, number of grains/ plant, grains weight/ spike and 100-grains weight under normal and water stress conditions.

\begin{tabular}{|c|c|c|c|c|c|c|c|c|c|c|c|c|c|c|}
\hline \multirow{2}{*}{ Traits } & \multirow{2}{*}{ Crosses } & \multirow{2}{*}{$\begin{array}{l}\text { Statistical } \\
\text { Parameter }\end{array}$} & \multicolumn{6}{|c|}{ Normal } & \multicolumn{6}{|c|}{ stress } \\
\hline & & & $P_{1}$ & $\mathrm{P}_{2}$ & $F_{1}$ & $\mathrm{~F}_{2}$ & $\mathrm{BC}_{1}$ & $\mathrm{BC}_{2}$ & $P_{1}$ & $\mathrm{P}_{2}$ & $F_{1}$ & $\mathrm{~F}_{2}$ & $\mathrm{BC}_{1}$ & $\mathrm{BC}_{2}$ \\
\hline \multirow{12}{*}{$\begin{array}{l}\text { Number of } \\
\text { spikes / plant } \\
\text { (spike) }\end{array}$} & \multirow{3}{*}{1} & $x^{-}$ & 15.50 & 16.60 & 19.30 & 17.28 & 13.64 & 17.80 & 8.30 & 14.00 & 14.50 & 13.53 & 8.08 & 12.51 \\
\hline & & $S^{2}$ & 1.91 & 0.87 & 1.04 & 23.18 & 14.99 & 20.89 & 1.25 & 1.86 & 1.50 & 19.98 & 18.32 & 16.33 \\
\hline & & $S^{2} x^{-}$ & 0.06 & 0.03 & 0.03 & 0.08 & 0.20 & 0.28 & 0.04 & 0.06 & 0.05 & 0.07 & 0.24 & 0.22 \\
\hline & \multirow{3}{*}{2} & $x^{-}$ & 14.90 & 16.00 & 18.40 & 16.47 & 14.00 & 16.00 & 12.50 & 14.20 & 15.90 & 13.89 & 11.32 & 14.07 \\
\hline & & $S^{2}$ & 4.02 & 4.14 & 4.80 & 45.32 & 33.97 & 35.73 & 2.33 & 0.99 & 1.13 & 13.22 & 10.68 & 11.25 \\
\hline & & $S^{2} x^{-}$ & 0.13 & 0.14 & 0.16 & 0.15 & 0.45 & 0.48 & 0.08 & 0.03 & 0.04 & 0.04 & 0.14 & 0.15 \\
\hline & \multirow{3}{*}{3} & $\mathrm{X}^{-}$ & 20.30 & 16.20 & 25.90 & 19.54 & 18.84 & 15.73 & 16.60 & 14.20 & 20.40 & 17.19 & 15.88 & 13.83 \\
\hline & & $S^{2}$ & 2.70 & 2.86 & 2.58 & 41.03 & 27.22 & 32.04 & 1.70 & 1.41 & 2.32 & 21.89 & 20.94 & 13.04 \\
\hline & & $S^{2} x^{-}$ & 0.09 & 0.10 & 0.09 & 0.14 & 0.36 & 0.43 & 0.06 & 0.05 & 0.08 & 0.07 & 0.28 & 0.17 \\
\hline & \multirow{3}{*}{4} & $x^{-}$ & 15.20 & 15.90 & 19.40 & 16.71 & 14.68 & 16.76 & 10.70 & 13.70 & 15.00 & 14.52 & 12.04 & 14.65 \\
\hline & & $\mathrm{S}^{2}$ & 2.44 & 1.75 & 1.49 & 37.83 & 29.90 & 28.73 & 2.08 & 1.87 & 1.66 & 22.81 & 21.04 & 17.18 \\
\hline & & $S^{2} x^{-}$ & 0.08 & 0.06 & 0.05 & 0.13 & 0.40 & 0.38 & 0.07 & 0.06 & 0.06 & 0.08 & 0.28 & 0.23 \\
\hline \multirow{12}{*}{$\begin{array}{l}\text { Number of } \\
\text { grains / spike } \\
\text { (grain) }\end{array}$} & \multirow{3}{*}{1} & $\mathrm{X}^{-}$ & 67.40 & 63.90 & 73.90 & 71.53 & 67.28 & 61.36 & 65.07 & 61.40 & 71.10 & 68.43 & 64.83 & 59.37 \\
\hline & & $S^{2}$ & 7.42 & 16.44 & 7.33 & 157.13 & 3113.88 & 123.88 & 4.34 & 14.32 & 5.06 & 143.36 & 120.17 & 102.37 \\
\hline & & $S^{2} x^{-}$ & 0.25 & 0.55 & 0.24 & 0.52 & 1.52 & 1.65 & 0.14 & 0.48 & 0.17 & 0.48 & 1.60 & 1.36 \\
\hline & \multirow{3}{*}{2} & $x^{-}$ & 67.47 & 65.80 & 68.10 & 66.55 & 65.43 & 64.21 & 60.80 & 64.90 & 67.70 & 65.85 & 58.12 & 63.04 \\
\hline & & $S^{2}$ & 8.12 & 5.96 & 4.85 & 153.22 & 2122.65 & 129.47 & 5.89 & 9.61 & 13.87 & 156.71 & 125.51 & 122.66 \\
\hline & & $S^{2} x^{-}$ & 0.27 & 0.20 & 0.16 & 0.51 & 1.64 & 1.73 & 0.20 & 0.32 & 0.46 & 0.52 & 1.67 & 1.64 \\
\hline & \multirow{3}{*}{3} & $x^{-}$ & 69.73 & 64.70 & 73.60 & 71.55 & 66.99 & 61.92 & 58.40 & 61.70 & 68.40 & 63.74 & 57.67 & 60.48 \\
\hline & & $S^{2}$ & 7.24 & 6.01 & 9.35 & 136.32 & 2103.12 & 110.75 & 9.77 & 7.25 & 5.42 & 130.50 & 102.06 & 105.31 \\
\hline & & $S^{2} x^{-}$ & 0.24 & 0.20 & 0.31 & 0.45 & 1.37 & 1.48 & 0.33 & 0.24 & 0.18 & 0.44 & 1.36 & 1.40 \\
\hline & \multirow{3}{*}{4} & $\mathrm{X}^{-}$ & 64.53 & 65.60 & 69.40 & 66.99 & 63.79 & 60.36 & 60.07 & 64.30 & 68.50 & 61.07 & 57.87 & 58.48 \\
\hline & & $S^{2}$ & 10.19 & 6.87 & 14.32 & 155.20 & 0105.87 & 127.69 & 4.96 & 6.42 & 3.16 & 139.65 & 110.82 & 107.06 \\
\hline & & $S^{2} x^{-}$ & 0.34 & 0.23 & 0.48 & 0.52 & 1.41 & 1.70 & 0.17 & 0.21 & 0.11 & 0.47 & 1.48 & 1.43 \\
\hline \multirow{12}{*}{$\begin{array}{c}\text { grains weight/ } \\
\text { spike } \\
\text { (gm) }\end{array}$} & \multirow{3}{*}{1} & $x^{-}$ & 3.22 & 2.47 & 4.11 & 3.84 & 3.04 & 2.90 & 2.96 & 2.11 & 3.29 & 2.89 & 2.96 & 2.45 \\
\hline & & $S^{2}$ & 0.04 & 0.03 & 0.05 & 0.74 & 0.55 & 0.55 & 0.05 & 0.01 & 0.05 & 0.74 & 0.55 & 0.55 \\
\hline & & $S^{2} x^{-}$ & 0.001 & 0.001 & 0.002 & 0.002 & 0.007 & 0.007 & 0.002 & 0.0003 & 0.002 & 0.002 & 0.007 & 0.007 \\
\hline & & $x^{-}$ & 3.28 & 3.39 & 4.06 & 3.61 & 3.31 & 3.35 & 2.66 & 3.01 & 3.62 & 2.82 & 2.41 & 3.07 \\
\hline & 2 & $S^{2}$ & 0.02 & 0.06 & 0.01 & 1.00 & 0.87 & 0.79 & 0.06 & 0.04 & 0.04 & 0.74 & 0.50 & 0.54 \\
\hline & & $S^{2} x^{-}$ & 0.001 & 0.002 & 0.0005 & 0.003 & 0.012 & 0.011 & 0.002 & 0.001 & 0.001 & 0.002 & 0.007 & 0.007 \\
\hline & & $x^{-}$ & 3.03 & 2.60 & 3.48 & 2.87 & 3.32 & 2.89 & 2.84 & 2.57 & 3.01 & 2.68 & 2.48 & 2.72 \\
\hline & 3 & $S^{2}$ & 0.03 & 0.04 & 0.05 & 0.69 & 0.46 & 0.49 & 0.04 & 0.06 & 0.05 & 0.51 & 0.35 & 0.39 \\
\hline & & $S^{2} x^{-}$ & 0.001 & 0.001 & 0.002 & 0.002 & 0.006 & 0.007 & 0.001 & 0.002 & 0.002 & 0.002 & 0.005 & 0.005 \\
\hline & & $\mathrm{x}^{-}$ & 3.72 & 3.53 & 4.21 & 3.94 & 3.09 & 2.94 & 2.89 & 2.79 & 3.31 & 3.08 & 2.52 & 2.59 \\
\hline & 4 & $S^{2}$ & 0.03 & 0.05 & 0.05 & 0.91 & 0.50 & 0.70 & 0.06 & 0.03 & 0.03 & 1.02 & 0.71 & 0.55 \\
\hline & & $S^{2} x^{-}$ & 0.001 & 0.002 & 0.002 & 0.003 & 0.007 & 0.009 & 0.002 & 0.001 & 0.001 & 0.003 & 0.009 & 0.007 \\
\hline & & $x^{-}$ & 4.76 & 4.46 & 5.73 & 5.16 & 4.82 & 4.84 & 4.62 & 4.09 & 5.09 & 4.89 & 4.75 & 4.26 \\
\hline & 1 & $S^{2}$ & 0.05 & 0.03 & 0.05 & 1.47 & 1.06 & 0.94 & 0.05 & 0.02 & 0.02 & 0.79 & 0.52 & 0.58 \\
\hline & & $S^{2} x^{-}$ & 0.002 & 0.001 & 0.002 & 0.005 & 0.014 & 0.013 & 0.002 & 0.001 & 0.001 & 0.003 & 0.007 & 0.008 \\
\hline & & $\mathrm{X}^{-}$ & 5.03 & 4.91 & 6.32 & 5.47 & 5.19 & 5.61 & 4.58 & 4.67 & 5.41 & 4.86 & 4.37 & 4.42 \\
\hline & 2 & $S^{2}$ & 0.05 & 0.05 & 0.08 & 1.06 & 1.01 & 0.82 & 0.06 & 0.05 & 0.07 & 1.02 & 0.50 & 1.42 \\
\hline weight & & $S^{2} x^{-}$ & 0.002 & 0.002 & 0.003 & 0.004 & 0.014 & 0.011 & 0.002 & 0.002 & 0.002 & 0.003 & 0.007 & 0.019 \\
\hline (g) & & $x^{-}$ & 4.86 & 4.60 & 6.06 & 5.21 & 5.11 & 4.62 & 4.59 & 4.50 & 4.21 & 3.88 & 4.07 & 3.74 \\
\hline & 3 & $s^{2}$ & 0.06 & 0.08 & 0.03 & 1.27 & 0.91 & 0.93 & 0.04 & 0.04 & 0.02 & 0.95 & 0.73 & 0.86 \\
\hline & & $S^{2} x^{-}$ & 0.002 & 0.003 & 0.001 & 0.004 & 0.012 & 0.012 & 0.001 & 0.001 & 0.001 & 0.003 & 0.010 & 0.011 \\
\hline & & $x^{-}$ & 5.21 & 5.10 & 5.86 & 5.34 & 4.74 & 5.08 & 4.73 & 4.54 & 5.37 & 4.95 & 4.35 & 4.55 \\
\hline & 4 & $S^{2}$ & 0.08 & 0.05 & 0.05 & 1.17 & 1.00 & 0.98 & 0.07 & 0.02 & 0.05 & 1.12 & 0.94 & 0.72 \\
\hline & & $S^{2} x^{-}$ & 0.003 & 0.002 & 0.002 & 0.004 & 0.013 & 0.013 & 0.002 & 0.001 & 0.002 & 0.004 & 0.012 & 0.010 \\
\hline
\end{tabular}


Table (4-e ): Mean $\left(X^{-}\right)$, variance $\left(s^{2}\right)$ and variance of mean $\left(S^{2} x^{-}\right)$of $P_{1}, P_{2}, F_{1}, F_{2}, B C_{1}$ and $\mathrm{BC}_{2}$ populations of four crosses for biological yield/ plant and grain yield /plant under normal and water stress conditions.

\begin{tabular}{|c|c|c|c|c|c|c|c|c|c|c|c|c|c|c|}
\hline \multirow{2}{*}{ Traits } & \multirow{2}{*}{ Crosses } & \multirow{2}{*}{$\begin{array}{l}\text { Statistical } \\
\text { Parameter }\end{array}$} & \multicolumn{6}{|c|}{ Normal } & \multicolumn{6}{|c|}{ stress } \\
\hline & & & $P_{1}$ & $P_{2}$ & $F_{1}$ & $\mathrm{~F}_{2}$ & $\mathrm{BC}_{1}$ & $\mathrm{BC}_{2}$ & $P_{1}$ & $P_{2}$ & $F_{1}$ & $F_{2}$ & $\mathrm{BC}_{1}$ & $\mathrm{BC}_{2}$ \\
\hline \multirow{12}{*}{$\begin{array}{l}\text { Biological } \\
\text { yield / plant } \\
\text { (g) }\end{array}$} & \multirow{3}{*}{1} & $x^{-}$ & 70.39 & 68.33 & 77.33 & 72.11 & 73.08 & 66.56 & 69.25 & 67.67 & 70.74 & 68.11 & 66.75 & 61.98 \\
\hline & & $S^{2}$ & 4.61 & 8.90 & 6.10 & 86.87 & 66.33 & 55.60 & 5.60 & 7.63 & 7.69 & 80.75 & 57.01 & 58.87 \\
\hline & & $S^{2} x^{-}$ & 0.15 & 0.30 & 0.20 & 0.29 & 0.88 & 0.74 & 0.19 & 0.25 & 0.26 & 0.27 & 0.76 & 0.78 \\
\hline & \multirow{3}{*}{2} & $x^{-}$ & 82.93 & 83.83 & 85.22 & 81.26 & 78.71 & 80.21 & 79.87 & 75.01 & 80.98 & 77.79 & 72.30 & 73.96 \\
\hline & & $S^{2}$ & 5.86 & 7.72 & 7.01 & 83.29 & 52.45 & 66.01 & 6.88 & 7.17 & 9.77 & 81.70 & 70.38 & 59.50 \\
\hline & & $S^{2} x^{-}$ & 0.20 & 0.26 & 0.23 & 0.28 & 0.70 & 0.88 & 0.23 & 0.24 & 0.33 & 0.27 & 0.94 & 0.79 \\
\hline & \multirow{3}{*}{3} & $x^{-}$ & 85.27 & 68.94 & 86.40 & 76.57 & 82.20 & 63.39 & 80.08 & 65.31 & 81.47 & 70.35 & 77.40 & 60.25 \\
\hline & & $S^{2}$ & 6.36 & 8.28 & 6.25 & 75.08 & 59.22 & 56.94 & 7.13 & 5.79 & 7.15 & 78.44 & 61.97 & 59.54 \\
\hline & & $S^{2} x^{-}$ & 0.21 & 0.28 & 0.21 & 0.25 & 0.79 & 0.76 & 0.24 & 0.19 & 0.24 & 0.26 & 0.83 & 0.79 \\
\hline & \multirow{3}{*}{4} & $x^{-}$ & 70.37 & 80.27 & 88.70 & 79.26 & 76.76 & 75.88 & 65.64 & 78.64 & 82.93 & 70.57 & 72.43 & 62.37 \\
\hline & & $S^{2}$ & 7.42 & 8.58 & 6.59 & 75.95 & 50.98 & 54.37 & 7.91 & 6.23 & 8.37 & 80.74 & 55.30 & 57.75 \\
\hline & & $S^{2} x^{-}$ & 0.25 & 0.29 & 0.22 & 0.25 & 0.68 & 0.72 & 0.26 & 0.21 & 0.28 & 0.27 & 0.74 & 0.77 \\
\hline \multirow{12}{*}{$\begin{array}{l}\text { Grain yield / } \\
\text { plant } \\
\text { (g) }\end{array}$} & \multirow{3}{*}{1} & $x^{-}$ & 22.70 & 23.95 & 35.38 & 15.38 & 25.83 & 19.11 & 19.56 & 16.60 & 28.90 & 20.45 & 15.69 & 17.72 \\
\hline & & $S^{2}$ & 1.41 & 3.25 & 2.30 & 67.07 & 62.98 & 45.38 & 3.15 & 5.21 & 5.72 & 85.82 & 65.19 & 62.66 \\
\hline & & $S^{2} x^{-}$ & 0.05 & 0.11 & 0.08 & 0.22 & 0.84 & 0.61 & 0.10 & 0.17 & 0.19 & 0.29 & 0.87 & 0.84 \\
\hline & \multirow{3}{*}{2} & $x^{-}$ & 30.96 & 27.97 & 34.14 & 25.01 & 26.72 & 24.70 & 25.37 & 21.74 & 29.73 & 23.51 & 22.57 & 20.00 \\
\hline & & $S^{2}$ & 3.78 & 3.90 & 6.54 & 76.54 & 64.02 & 50.64 & 4.31 & 5.32 & 7.65 & 84.16 & 65.45 & 66.20 \\
\hline & & $S^{2} x^{-}$ & 0.13 & 0.13 & 0.22 & 0.26 & 0.85 & 0.68 & 0.14 & 0.18 & 0.26 & 0.28 & 0.87 & 0.88 \\
\hline & \multirow{3}{*}{3} & $x^{-}$ & 29.65 & 19.94 & 33.25 & 24.71 & 33.44 & 20.95 & 34.08 & 12.38 & 12.30 & 18.63 & 18.39 & 17.66 \\
\hline & & $S^{2}$ & 4.67 & 4.41 & 3.85 & 77.78 & 66.37 & 54.37 & 3.35 & 3.57 & 6.83 & 79.25 & 67.42 & 45.91 \\
\hline & & $S^{2} x^{-}$ & 0.16 & 0.15 & 0.13 & 0.26 & 0.88 & 0.72 & 0.11 & 0.12 & 0.23 & 0.26 & 0.90 & 0.61 \\
\hline & \multirow{3}{*}{4} & $x^{-}$ & 23.80 & 26.39 & 32.78 & 28.83 & 24.39 & 23.28 & 20.38 & 22.08 & 30.08 & 25.50 & 21.40 & 19.69 \\
\hline & & $S^{2}$ & 3.50 & 4.90 & 6.87 & 79.62 & 63.31 & 58.41 & 4.11 & 5.51 & 8.40 & 93.31 & 64.31 & 67.92 \\
\hline & & $S^{2} x^{-}$ & 0.12 & 0.16 & 0.23 & 0.27 & 0.84 & 0.78 & 0.14 & 0.18 & 0.28 & 0.31 & 0.86 & 0.91 \\
\hline
\end{tabular}

Cross 1 (Giza $126 \times$ Giza 129), Cross2 (Giza $126 \times$ Giza 131), Cross 3 (Giza $131 \times$ Giza 129) and cross 4 (CC89 x Giza131)

or plant height, the data presented in Table $5-\mathrm{a}$ indicated that, the second and the third crosses exposed highly significant mid and better parental heterosis due to over-dominance (PR > $+1)$ under both conditions, while the first and the fourth crosses revealed highly significant mid and better parental heterosis under water stress condition and significant or highly significant over mid parent heterosis under normal condition a result of over-dominance in all cases.

The data in Table 5-a and Table 5-b pointed out that, the first, the second, the third and the fourth crosses revealed highly significant mid and better parental heterosis for spike length, peduncle length and no.of grains and weight per spike under both conditions due to over - dominance,in all crosses for the traits in consideration, with few exceptions i.e, mid parental heterosis under normal condition; better parent heterosis at stress condition in the first cross and better parent heterosis in the fourth cross under stress condition for peduncle length, where this values were only significant. Moreover, in the case of better parent heterosis at normal condition for no.of grains/spike where the heterotic effect did'nt reach to the level of significant. 
Table (5-a) : Heterosis, potence ratio and inbreeding depression in four crosses for days to heading, days to maturity, filling period, filling rate, plant height, spike length, peduncle length, number of tillers/ plant and number of spike/ plant, under normal and water stress conditions.

\begin{tabular}{|c|c|c|c|c|c|c|c|c|c|}
\hline \multirow{3}{*}{ Traits } & \multirow{3}{*}{ Crosses } & \multicolumn{4}{|c|}{ Normal } & \multicolumn{4}{|c|}{ Stress } \\
\hline & & \multirow{2}{*}{$\frac{\text { Heterosis }}{\mathrm{MP}}$} & \multicolumn{3}{|c|}{$\underline{\text { Heterosis }}$} & \multirow{2}{*}{$\frac{\text { Heterosis }}{\mathrm{MP}}$} & \multicolumn{3}{|c|}{ Hetrosis } \\
\hline & & & PR & $\overline{\mathrm{BP}}$ & ID\% & & PR & $\mathrm{BP}$ & ID\% \\
\hline \multirow{4}{*}{$\begin{array}{l}\text { Days to } \\
\text { heading }\end{array}$} & 1 & $1.95^{\star *}$ & 2.54 & $2.74^{\star \star}$ & 0.56 & $3.38^{* *}$ & 2.04 & $5.12^{* *}$ & $3.40^{\star *}$ \\
\hline & 2 & $-2.72^{* \star}$ & -3.29 & $-1.91^{* *}$ & $0.95^{\star *}$ & $0.81^{\star *}$ & 4.33 & $1.00^{\star \star}$ & $3.72^{\star \star}$ \\
\hline & 3 & $-3.10^{* \star}$ & -10.60 & $-2.82^{\star *}$ & $-0.75^{\star}$ & $0.49^{*}$ & 0.33 & $1.99^{* *}$ & $1.87^{\star *}$ \\
\hline & 4 & $-4.14^{\star \star}$ & -5.83 & $-3.46^{\star *}$ & -0.40 & $-2.97^{\star \star}$ & -2.76 & $-1.92^{\star *}$ & $-5.27^{\star \star}$ \\
\hline \multirow{4}{*}{$\begin{array}{l}\text { Days to } \\
\text { maturity }\end{array}$} & 1 & $3.81^{* *}$ & 1.89 & $5.94^{* *}$ & $2.80^{\star \star}$ & $-0.61^{* *}$ & -0.35 & $1.15^{\star \star}$ & $-0.91^{\star \star}$ \\
\hline & 2 & $2.88^{\star \star}$ & 2.62 & $4.03^{\star \star}$ & $3.05^{\star *}$ & $0.30^{*}$ & 0.37 & $1.12^{\star \star}$ & $1.51^{\star *}$ \\
\hline & 3 & $-0.97^{\star *}$ & -0.58 & $0.70^{\star *}$ & $-0.98^{\star *}$ & $-0.68^{* *}$ & -0.44 & $0.88^{* *}$ & $-0.39^{*}$ \\
\hline & 4 & $-1.69^{\star \star}$ & -2.64 & $-1.06^{* *}$ & $-0.82^{* *}$ & $-1.35^{\star *}$ & -2.46 & $-0.81^{* *}$ & $-1.14^{\star *}$ \\
\hline \multirow{4}{*}{$\begin{array}{l}\text { Grain filling } \\
\text { period }\end{array}$} & 1 & $5.00^{* *}$ & 0.44 & $18.38^{\star *}$ & $7.51^{\star *}$ & $-10.10^{* *}$ & -1.03 & -0.32 & $-4.36^{\star *}$ \\
\hline & 2 & $7.94^{\star *}$ & 2.50 & $11.48^{\star *}$ & $7.40^{* *}$ & -1.23 & -0.43 & $1.69^{*}$ & 1.05 \\
\hline & 3 & $-5.17^{\star \star}$ & -0.58 & $4.10^{\star *}$ & $-6.09^{\star *}$ & $-3.02^{\star *}$ & -0.37 & $5.52^{* \star}$ & -1.05 \\
\hline & 4 & $-9.34^{* *}$ & -1.94 & $-4.74^{\star *}$ & 0.03 & $-8.65^{\star *}$ & -2.47 & $-5.35^{\star *}$ & $-3.45^{\star *}$ \\
\hline \multirow{4}{*}{$\begin{array}{l}\text { Grain filling } \\
\text { rate }\end{array}$} & 1 & $41.79^{\star \star}$ & 3.11 & $25.00^{* *}$ & $51.58^{\star *}$ & $77.36^{* *}$ & 41.00 & $74.07^{\star *}$ & $46.81^{* *}$ \\
\hline & 2 & $44.87^{\star \star}$ & 35.00 & $43.04^{\star *}$ & $38.94^{\star *}$ & $28.13^{\star \star}$ & 6.00 & $22.39^{* *}$ & $40.24^{* *}$ \\
\hline & 3 & $13.48^{\star *}$ & 0.46 & $-12.17^{\star *}$ & $26.73^{\star \star}$ & $-43.28^{\star *}$ & -1.07 & $-59.57^{\star *}$ & $-52.63^{\star *}$ \\
\hline & 4 & $27.03^{\star *}$ & 1.33 & $5.62^{*}$ & $9.57^{\star *}$ & $22.22^{* *}$ & 1.50 & $6.45^{\star}$ & $-27.27^{\star *}$ \\
\hline \multirow{4}{*}{ Plant height } & 1 & $1.59^{\star \star}$ & 1.23 & 0.29 & $1.78^{\star \star}$ & $202^{* *}$ & 3.33 & $1.4^{\star *}$ & 0.33 \\
\hline & 2 & $6.26^{\star *}$ & 3.32 & $4.29^{\star *}$ & $1.51^{*}$ & $8.92^{* *}$ & 15.73 & $8.31^{* *}$ & $1.38^{*}$ \\
\hline & 3 & $3.69^{\star *}$ & 18.50 & $3.49^{\star \star}$ & $2.45^{\star *}$ & $2.34^{\star \star}$ & 5.75 & $1.92^{*}$ & 1.24 \\
\hline & 4 & $0.92^{* *}$ & 2.71 & 0.58 & 1.08 & $4.93^{\star *}$ & 13.86 & $4.56^{\star \star}$ & 1.39 \\
\hline \multirow{4}{*}{ Spike length } & 1 & $29.19^{* *}$ & 5.22 & $22.35^{\star *}$ & $7.50^{* *}$ & $38.69^{* *}$ & 7.57 & $31.94^{\star *}$ & $26.11^{* *}$ \\
\hline & 2 & $17.31^{* *}$ & 1.40 & $4.36^{\star *}$ & $18.25^{\star *}$ & $40.58^{* *}$ & 9.33 & $34.72^{* *}$ & $16.60^{* *}$ \\
\hline & 3 & $8.92^{\star \star}$ & 47.50 & $8.72^{\star \star}$ & $15.34^{\star *}$ & $11.15^{\star \star}$ & 12.35 & $10.21^{\text {** }}$ & $9.17^{\star *}$ \\
\hline & 4 & $28.21^{\star *}$ & 1.90 & $11.65^{\star \star}$ & $18.61^{\star *}$ & $25.88^{* *}$ & 2.00 & $11.46^{\star \star}$ & $17.01^{* *}$ \\
\hline \multirow{4}{*}{$\begin{array}{l}\text { Peduncle } \\
\text { Length }\end{array}$} & 1 & $30.97^{\star}$ & 10.47 & $27.20^{\star \star}$ & $26.90^{* *}$ & $14.08^{\star \star}$ & 1.32 & $3.07^{\star}$ & $4.94^{\star *}$ \\
\hline & 2 & $16.67^{* *}$ & 4.80 & $12.75^{\star *}$ & $4.67^{* *}$ & $12.08^{* *}$ & -6.40 & $10.00^{* *}$ & $10.51^{\star *}$ \\
\hline & 3 & $20.33^{\star *}$ & 5.82 & $16.27^{\star \star}$ & $15.39^{* \star}$ & $19.82^{* *}$ & 7.50 & $16.74^{\star *}$ & $15.33^{* *}$ \\
\hline & 4 & $14.65^{\star *}$ & -3.24 & $9.69^{* *}$ & $5.27^{* *}$ & $9.16^{* *}$ & -1.35 & $2.24^{*}$ & $6.72^{* *}$ \\
\hline \multirow{4}{*}{$\begin{array}{l}\text { Number of } \\
\text { tillers / plant }\end{array}$} & 1 & $16.38^{\star \star}$ & 3.19 & $10.70^{\star \star}$ & $12.44^{* *}$ & $34.55^{\star \star}$ & 1.17 & $3.93^{*}$ & $36.24^{\star *}$ \\
\hline & 2 & $34.03^{* *}$ & 3.54 & $22.27^{\star \star}$ & $13.41^{* \star}$ & $15.25^{\star *}$ & 4.09 & $11.11^{\star *}$ & 1.35 \\
\hline & 3 & $19.71^{\star *}$ & 1.29 & $3.80^{*}$ & $25.16^{\star \star}$ & $-10.28^{\star *}$ & -1.14 & $-17.71^{\star *}$ & $18.89^{* *}$ \\
\hline & 4 & $9.33^{\star *}$ & 1.50 & 2.93 & $9.34^{* *}$ & $18.73^{\star *}$ & 65.00 & $18.39^{* *}$ & $11.89^{\star *}$ \\
\hline \multirow{4}{*}{$\begin{array}{l}\text { Number of } \\
\text { spikes / plant }\end{array}$} & 1 & $20.25^{\star \star}$ & 5.91 & $16.27^{\star \star}$ & $10.47^{\star \star}$ & $30.04^{\star *}$ & 1.18 & 3.57 & $6.69^{\star \star}$ \\
\hline & 2 & $19.09^{* *}$ & 5.36 & $15.00^{\star \star}$ & $10.49^{* \star}$ & $19.10^{\star *}$ & 3.00 & $11.97^{\star *}$ & $12.64^{\star *}$ \\
\hline & 3 & $41.92^{\star \star}$ & 3.73 & $27.59^{\star \star}$ & $24.56^{\star \star}$ & $32.47^{\star *}$ & 4.17 & $22.89^{\star *}$ & $15.74^{\star *}$ \\
\hline & 4 & $24.76^{\star \star}$ & 11.00 & $22.01^{\text {** }}$ & $13.87^{\star \star}$ & $22.95^{\star *}$ & 1.87 & $9.49^{* *}$ & 3.20 \\
\hline
\end{tabular}

$\left({ }^{*}\right)$ and $\left({ }^{* *}\right)$ significant at 0.05 and 0.01 levels probability, respectively.

Cross 1 (Giza 126 x Giza 129), Cross2 (Giza 126 x Giza 131), Cross 3 (Giza 131 x Giza 129) and cross 4 (CC89 x Giza131) 
Table (5-b) : Heterosis, potence ratio and inbreeding depression in four crosses for number of grains/ plant, grains weight/ spike, 100- grains weight, biological yield/ plant and grain yield/ plant under normal and water stress conditions.

\begin{tabular}{|c|c|c|c|c|c|c|c|c|c|}
\hline \multirow{3}{*}{ Traits } & \multirow{3}{*}{ Crosses } & \multicolumn{4}{|c|}{ Normal } & \multicolumn{4}{|c|}{ Stress } \\
\hline & & \multirow{2}{*}{$\frac{\text { Heterosis }}{\overline{\mathrm{MP}}}$} & \multicolumn{3}{|c|}{ Heterosis } & \multirow{2}{*}{$\frac{\text { Heterosis }}{\overline{\mathrm{MP}}}$} & \multicolumn{3}{|c|}{ Hetrosis } \\
\hline & & & PR & $\overline{\mathrm{BP}}$ & ID\% & & PR & $\mathrm{BP}$ & ID\% \\
\hline \multirow{4}{*}{$\begin{array}{l}\text { Number } \\
\text { of grains / } \\
\text { spike }\end{array}$} & 1 & $12.57^{\star *}$ & 4.71 & $9.64^{\star *}$ & 3.21 & $12.45^{\star *}$ & 4.29 & $9.27^{* \star}$ & 3.76 \\
\hline & 2 & $2.20^{\star *}$ & 1.76 & 0.93 & 2.28 & $7.72^{\star *}$ & 2.37 & $4.31^{* *}$ & 2.73 \\
\hline & 3 & $9.49^{* \star}$ & 2.54 & $5.55^{\star *}$ & 2.79 & $13.91^{* *}$ & 5.06 & $10.86^{\star *}$ & 6.82 \\
\hline & 4 & $6.65^{\star *}$ & 8.13 & $5.79^{\star *}$ & 3.47 & $10.16^{\star \star}$ & 2.99 & $6.53^{\star *}$ & 10.85 \\
\hline \multirow{4}{*}{$\begin{array}{l}\text { grains } \\
\text { weight/ } \\
\text { spike }\end{array}$} & 1 & $44.72^{\star \star}$ & 3.39 & $27.64^{\star \star}$ & $6.57^{* *}$ & $29.53^{\star *}$ & 1.76 & $11.15^{\star \star}$ & $12.16^{* *}$ \\
\hline & 2 & $21.92^{\star *}$ & 13.27 & $19.76^{* *}$ & $11.08^{\star *}$ & $27.92^{\star \star}$ & 4.51 & $20.27^{* *}$ & $22.10^{* *}$ \\
\hline & 3 & $23.84^{\star *}$ & 3.05 & $14.85^{\star *}$ & $17.53^{* *}$ & $11.48^{\star \star}$ & 2.30 & $5.99^{* *}$ & $10.96^{* *}$ \\
\hline & 4 & $15.98^{* *}$ & 6.11 & $13.17^{\star \star}$ & $6.41^{* *}$ & $16.55^{\star \star}$ & 9.40 & $14.53^{\star \star}$ & $6.65^{\star *}$ \\
\hline \multirow{4}{*}{$\begin{array}{c}100- \\
\text { grain } \\
\text { weight }\end{array}$} & 1 & $24.30^{\star *}$ & 7.47 & $20.38^{\star *}$ & $9.95^{\star \star}$ & $16.74^{\star \star}$ & 2.75 & $10.17^{\star \star *}$ & $3.93^{* \star}$ \\
\hline & 2 & $27.16^{* *}$ & 22.50 & $25.65^{\star \star}$ & $13.45^{\star *}$ & $17.10^{\star \star}$ & 17.56 & $15.85^{\star \star}$ & $10.17^{* *}$ \\
\hline & 3 & $28.12^{\star *}$ & 10.23 & $24.69^{\star \star}$ & $14.03^{\star *}$ & $-7.47^{\star \star}$ & -7.56 & $-8.28^{\star \star}$ & $7.84^{\star \star}$ \\
\hline & 4 & $14.90^{\star *}$ & 13.82 & $12.48^{\star *}$ & $8.87^{\star *}$ & $15.73^{\star *}$ & 7.68 & $13.53^{\star *}$ & $7.82^{\star \star}$ \\
\hline \multirow{4}{*}{$\begin{array}{l}\text { Biological } \\
\text { yield/plant }\end{array}$} & 1 & $11.49^{\star *}$ & 7.74 & $9.86^{\star \star}$ & $6.76^{\star *}$ & $3.33^{\star \star}$ & 2.89 & $2.15^{\star}$ & $3.72^{\star \star}$ \\
\hline & 2 & $2.24^{* *}$ & 4.09 & 1.66 & $4.65^{\star *}$ & $4.57^{\star \star}$ & 1.46 & 1.39 & $3.94^{* *}$ \\
\hline & 3 & $12.05^{\star *}$ & 1.14 & 1.33 & $11.38^{* *}$ & $12.08^{\star \star}$ & 1.19 & $1.74^{*}$ & $13.65^{\star *}$ \\
\hline & 4 & $17.76^{* *}$ & 2.70 & $10.50^{\star *}$ & $10.64^{\star *}$ & $14.96^{\star \star}$ & 1.66 & $5.46^{* *}$ & $14.90^{* *}$ \\
\hline \multirow{4}{*}{$\begin{array}{l}\text { Grain } \\
\text { yield / } \\
\text { plant }\end{array}$} & 1 & $51.68^{\star *}$ & 19.29 & $47.72^{\star \star}$ & $56.53^{\star *}$ & $59.85^{\star \star}$ & 7.31 & $47.75^{\star \star}$ & $29.24^{\star *}$ \\
\hline & 2 & $15.85^{\star *}$ & 3.12 & $10.27^{\star *}$ & $26.74^{\star \star}$ & $26.19^{\star \star}$ & 3.40 & $17.19^{\star *}$ & $20.92^{* *}$ \\
\hline & 3 & $34.07^{* *}$ & 1.74 & $12.14^{\star *}$ & $25.68^{\star *}$ & $-47.05^{\star *}$ & -1.01 & $-63.91^{* *}$ & $-51.46^{\star *}$ \\
\hline & 4 & $30.60^{* *}$ & 5.93 & $24.21^{\star *}$ & $12.05^{\star \star}$ & $41.69^{\star \star}$ & 10.41 & $36.23^{\star *}$ & $15.23^{* *}$ \\
\hline
\end{tabular}

$\left(^{*}\right)$ and $\left(^{* *}\right)$ significant at 0.05 and 0.01 levels probability, respectively.

Cross 1 (Giza 126 x Giza 129), Cross2 (Giza 126 x Giza 131), Cross 3 (Giza 131 x Giza 129) and cross 4 (CC89 x Giza131)

The data shown in Table 5-b illustrated that, highly significant mid and better parent heterosis in positive direction were obtained in the crosses; 1,2 and 4 for number of tillers/plant under both conditions, as a result of over- dominance in all cases, except in the fourth cross under normal condition where the heterotic effect over better parent was not significant, as a result of overdominance in all cases. Mean while, the third cross under normal condition had significant mid and better parent heterosis, as a result of over- dominance also.

For number of spikes/plant, all crosses exhibited mid and better parental heterosis under both conditions due to overdominance $(P R>+1)$, except in the first cross at stress condition were better parent heterosis was not significant.

The data shown in Table 5-b showed that, highly significant mid and better parent heterosis in positive direction were obtained in the crosses; 1,2 and 4 for 100-grain weight and grain yield/plant under both conditions, as a result of over- dominance in all cases. While, the third cross under normal condition had highly significant mid and better parent heterosis, due to overdominance also. 
For biological yield/ plant, the first and the fourth crosses exhibited significant mid and better parental heterosis under both conditions due to over-dominance (PR $>+1)$, while the second and the third crosses had highly significant mid parental heterosis under normal condition and better parental heterosis for the third cross under water stress condition. However, it could be concluded that, the fourth cross (CC89 $x$ Giza 131) had highly significant better parent heterosis-which considered as useful heterosis from the breeders point of viewunder both conditions for all studied traits, except for plant height and no.of tillers/plant at normal condition; the first cross (Giza 126 $x$ Giza 129) for grain filling rate, spike length, peduncle length, number of tillers/ plant, number of grains/spike, grains weight/spike, 100- grain weight, biological yield/plant and grain yield/plant under both conditions; the second cross (Giza 126 x Giza 131) for grain filling rate, plant height, spike length, peduncle length, number of tillers/ plant, number of spikes/plant, grains weight/spike, 100- grain weight and grain yield/plant under both conditions and the third cross (Giza 131 $x$ Giza 129) for plant height, spike length, peduncle length, number of spikes/plant, number of grains/spike and grains weight/spike under both conditions. These crosses could be used in suitable breeding programs aiming to improve barley either at normal irrigation or stress condition. Moreover, the heterotic effects in these crosses mostly attributed to over-dominance which helps the breeder to discover a transgresive segregation in early generations. El-Shawy (2008), Khattab et al. (2010) and El-Akhdar (2011) obtained overdominance prevailing in most crosses.

Inbreeding depression were found to be highly significant in positive direction for no.of days to heading and maturity in the second cross under both environmental conditions which means that $F_{2}$ population in theses cases was more earlier than that of $F_{1}, \mathrm{~s}$. The same direction was observed for days to heading at stress condition in the first and third crosses; and for days to maturity and grain filling period in the first cross at normal condition. However, the presence in vigor in F2 could be arributed to additive and epistatic gene action. Such crosses for these traits are excepted to give segregates superior to the better parent in these traits, which may be handled through pedigree method.

On the other hand, inbreeding depression values were found to be significant and/or highly significant in positive direction for grain filling rate, spike length, peduncle length, no.of tillers/ plant, no.of spikes/plant, grains weight/spike, 100grain weight, biological yield/plant and grain yield/plant in all crosses under both environmental conditions, with some few exceptions i.e., in the third and fourth crosses at stress condition for grain filling rate; the second cross no.of tillers/ plant; in the fourth cross at stress condition for no.of spikes/plant and the third cross at stress condition for grain yield/plant, were the values of inbreeding depression were highly significant in negative direction or insignificant values. However in the first case the expression of heterosis in the $F_{1}$ followed by a respectively reduction in $F_{2}$ would be attributed to the direct effect of homozygosity which was in harmony with the results obtained by Mahmoud Badeaa (2006), Abd-El-Haleem et al. (2010) in cotton, Khattab et al. (2010) and El-Akhdar (2011). While in the excepted cases, low inbreeding depression might suggests that, increasing retention in vigor in $\mathrm{F} 2$ is expected to be mainly due to accumulation of favorable additive genes (Shukla and Gautam (1990).

\section{REFERENCES}

Abd- El-Haleem, S.H.M., E M.R. Metwali and A.M.M. Al-Felaly (2010). Genetic analysis of yield and its components of some Egyptian cotton (Gossypium barbadense L.) varieties. World Journal of Agricultural Sci., 6 (5): 615-621. 
Amer, Kh. A. (2010). Inheritance of drought tolerance in some barley genotypes. Egypt. J. Agric., Res., 88(1): 85-102.

Amer, KH.A. (2011). Genetic analysis of yield and its components under normal and drought conditions in some barley crosses. Egypt. J. PI. Breed 15 (2):37-56.

Eid, A. A. (2006). Breeding studies on some barley diseases. Ph.D. Thesis, Fac. Agric. Menofiya. Univ. Egypt.

El-Akhdar, A. A. A. (2011). Genetic studies on yield and its components in some barley crosses. M.Sc. Thesis Fac., Agric., Kafrelsheikh, Uni., Egypt.

El-Bawab, A. M. O. (2003). Genetic studies on some characters in barley. Egypt. J. Agric., 81(2):235-255.

El-Sayed, M. M. A. (2007). Estimation of quantitative genetic statistics in diallel crosses of barley. M.Sc. Thesis Fac., Agric., Kafrelsheikh, Univ., Egypt.

El-Shawy, E. E. A. (2008). Genetic analysis of some important traits of six-rowed barley in normal and saline affected fields. M.Sc. Thesis Fac., Agric., Kafrelsheikh, Univ., Egypt.

Gamble, E.E. (1962). Gene effects in corn (Zea mays L.). I-Separtion and relative importance of gene effects for yield. Can. J. Plant Sci., 42: 339-348.

Katta, Y.S., A.A. Eid., M.S. Abd El-Aty and Sally, M. EL-Wakeel (2009). Studies on tolerance of some hulless barley crosses to drought. $6^{\text {th }}$ international plant breeding conf., Ismalia, Egypt. May-5-5: (867-885).

Khattab S. A. M., R. M. Esmail and Abd ElRahman M. F. Al-Ansary (2010). Genetical analysis of some quantitative traits in bread weight (Triticum aestivum L.). New York Science Journal 3(11): 152-157.

Mahmoud, Badeaa. A. M. (2006). Genetic evaluation of some barley traits in crosses under saline and non-saline conditions. M. Sc. Thesis Fac., Agric., Kafr El-Sheikh, Tanta Univ., Egypt.

Mather, K. and J.L. Jinks (1982). In Biometrical Genetics, third ed.

Mohamed, N. E. M. (2014). Genetic control for some traits using generation mean analysis in bread wheat (Triticum aestivum L.). International Journal of Plant and Soil Science 3(9): 1055-1068, 2014; Article no. IJPSS.

Simth, H. H. (1952). Fixing transgressive vigor in Nicotiana rustica. In Gowen Heterosis, lowa state College Press. Ames, la, U. S. A.

Shukla, A.K and N.C. Gautam (1990). Heterosis and inbreeding depression in okra (Abdelmoschus esculentus L. Moench) Indian J. Hortic., 47 : 85-88. 
تقدير قوة الهجين والتريبه الداخليه فى الثعير تحت ظروف الرى الطبيعى والإجهاد المائى رمضان علي الرفاعي(1) ، السيد حامد الصعيدى(1) ، أمجدعبدالغفار الجمال(1) ، محمد منصور

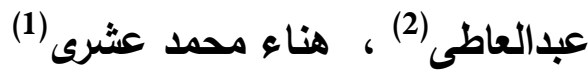
(1) قسم المحاصيل - كلية الزراعه - جامعة طنطا. (2) (2) قسم بحوث الثنعير - معهد بحوث المحاصيل - الحقليه - مركز البحوث الزراعيه.

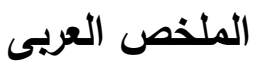
أجريت هذه الدراسة بمزرعة محطة البحوث الزراعبه بسخا - كفرالثيخ خلال ثلاث مواسم 2014/2013،

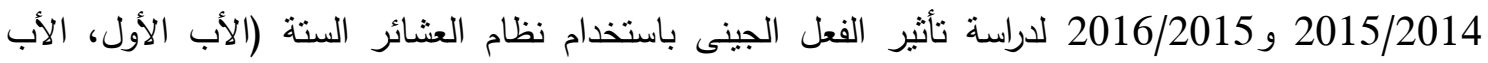

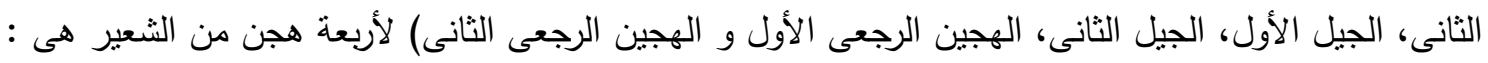

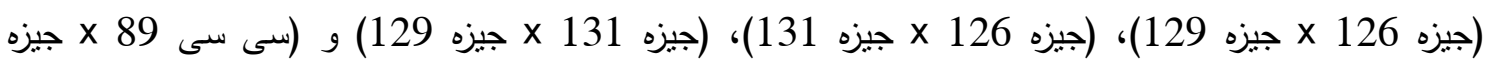

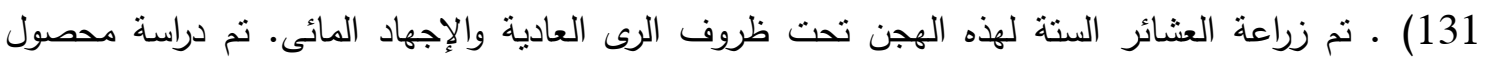

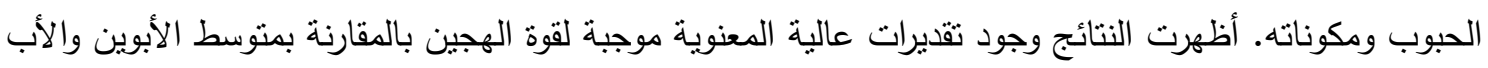

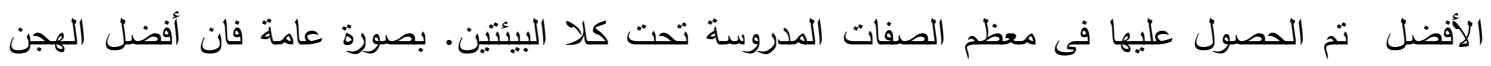

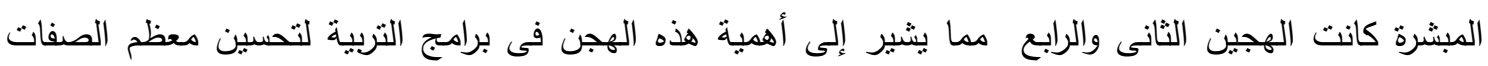

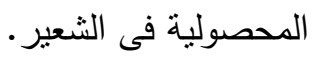



Menoufia J. Plant Prod., Vol. 2 February (2017): 39 - 51

$-1-$ 

Menoufia J. Plant Prod., Vol. 2 February (2017): 39 - 51

$-1-$ 